\title{
Data review processes in the analytical laboratory
}

\author{
Luiza Gimenes Rodrigues Albuquerque
}

January 2020

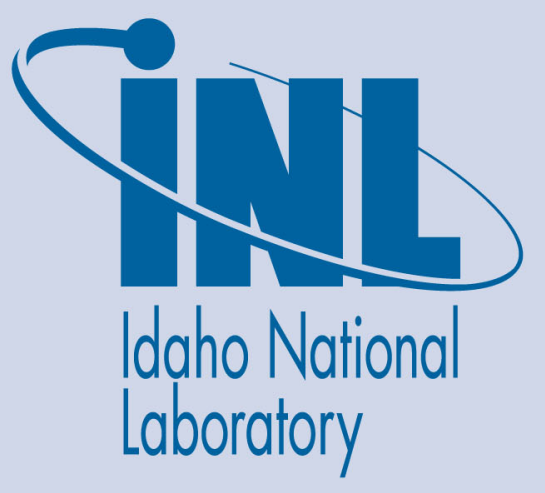

The INL is a U.S. Department of Energy National Laboratory operated by Battelle Energy Alliance 


\title{
Data review processes in the analytical laboratory
}

\author{
Luiza Gimenes Rodrigues Albuquerque
}

January 2020

Idaho National Laboratory Idaho Falls, Idaho 83415

http://www.inl.gov

Prepared for the U.S. Department of Energy Office of Nuclear Energy Under DOE Idaho Operations Office Contract 103190RSF 
Checklist for alpha spectrometry counting data review:

1) Check Sample ID

2) Alpha Vision v6.0

a. Check that the Tracer matches what our narrative states we traced with. The printout will give a percent recovery which should be between $45-110 \%$

b. Check under general analysis that the correct library was used (for example, if the analysis was for Uranium isotopes, ensure the Uranium regions of interest are shown on the spectrum,

c. Check sample size.

d. Ensure background and calibration dates in the Acquisition portion of the printout are before the Acquisition start date.

3) Check how well data agrees with gamma, AES, Q-ICP-MS or MC-ICP-MS, etc.

4) Check that data was uploaded correctly into LIMS and reflect "final" values.

a. Final values can be found in the Excel spreadsheet under "Reporting ALL in DPM".

5) Check that the data is consistent with what you know about the sample

a. If you can't answer this question, then find out more about the sample or compare with older results

6) Check that the data meets the requirements and goals of the customer

a. Did we meet detection limits?

b. Did we meet uncertainty requirements?

c. Did we measure and report all of the required data?

d. Did we measure analytes not requested by the customer? If so think about reporting them. 


\section{Checklist for CSONH data:}

1) Were the appropriate base calibration constants used?

2) Was the calibration constant calculated correctly and is it between $0.5-2$ ?

3) Is the base and calibration constant calculated for the correct channel? (i.e. low C vs. high C)

4) Were the blank areas consistent and reasonable throughout the runs?

a) Are the blank peak areas similar to previous runs with similar settings?

b) Was there significant drift in the peak areas throughout the measurements?

i) If so, was this accounted for in the uncertainty?

5) Was the check standard run in triplicate for each channel being calibrated?

6) Were the calibration standard results within the certified $2 \sigma$ Uncertainty.

a) If one failed, is it identified and noted why on the spreadsheet?

b) Are the calibration standard concentration and uncertainty identified on the spreadsheet?

7) Were the check standard results within the certified $2 \sigma$ uncertainty?

a) If not, can the error be increased within the bounds of the program's requirements?

b) If not, are the peak areas similar to previous runs with the same standards?

c) Are the check standard concentrations and uncertainty identified on the spreadsheet?

8) Was a check standard run before and after the samples, at a minimum?

a) If not, is it noted why on the spreadsheet?

9) Are the LOQ's calculated for each element measured?

a) Do the LOQ's meet the program requirements?

b) If 15 times the standard deviation is too high, talk to the program about using 10 times.

10) Are the sample weights in the spreadsheet entered correctly?

a) Check them against the log book and the safeguards tracking data if needed.

11) Is the uncertainty of the weights accounted for correctly?

a) Samples that are weighed by difference, such as in a dish, should have a higher uncertainty than those weighed directly on the balance.

12) Is the data summarized in an easily identified table at the bottom of the spreadsheet?

a) Does it include the AL\#'s, weight, elemental concentrations, and errors for each?

b) Are the units of concentration in ppm and mass in grams?

c) Is the weight error reported in grams and the concentration error in relative \%?

13) Was the data put into the GUM template to calculate the total propagated uncertainty?

a) Do the results calculated in the software match the results in the spreadsheet?

b) Was the error transferred from GUM to the spreadsheet correctly?

14) Is the appropriate number of significant figures displayed in the results?

15) Has the data been entered into LIMS correctly?

16) Have the data spreadsheet and GUM worksheets been sent to the ALDATA Group? 


\section{Checklist for gamma data review (Apex-Gamma report):}

\section{1) Gamma Spectrum Analysis:}

a. Check Sample ID

b. Check Sample Type and Sample Size.

i. If described as " $1 \mathrm{~mL}$ scint vial", sample size should be $1 \mathrm{~mL}$.

ii. This can vary for other samples types/sizes, and is where the correction for sample size is entered (for example, if a 1:100 dilution is counted, this is what corrects the results back to per gram of sample).

c. Check Dead Time

i. Should generally be $<15 \%$, although there are (rare) exceptions where the sample can't be diluted or moved farther from the detector to reduce the dead time below $15 \%$. This is most commonly found in hot cell cans.

\section{2) Peak Analysis report}

a. Check the Full Width at Half Maximum (FWHM)

i. Should be between 1 and 3 (not much higher than 3 )

\section{3) Background Subtract Report}

a. Subtracted Uncertainty has a relative uncertainty of less than $50 \%$ of the Subtracted Area

b. Compare the Subtracted area with the Original Area. If they differ significantly, chances are that the peak ID a false positive. Check the Subtracted Uncertainty again to be sure

c. Compare the Ambient Background with the Original Area. If they are similar, chances are that the peak is a false positive

\section{4) Nuclide Identification Report}

a. Check the ID confidence. Values close to 1 mean that there are high chances that the nuclide was correctly identified by the software

b. Check the Activity and Activity Uncertainty. The uncertainty should be at least $50 \%$ smaller than the activity

c. Check if different Energies (i.e., gamma lines) for the same nuclide gives similar activities.

i. If not, check if it is an energy with low yield. This means that the evaluated line is not as reliable as lines with higher yields

ii. If the same energy is identified as being a component for multiple isotopes and both identifications are accurate, this could result in a larger variation in activities of energies in the same nucleus than expected.

d. If, for a certain nuclide, an energy with a lower yield was identified but one energy with a higher yield was not, this can be an indicative of misidentification.

i. For peaks that you are not sure why they were identified, look at the nuclide decay chart. If you see some of the daughter of that decay chain, others might be identified as well, even with low ID confidence.

\section{5) Interference corrected report}

a. Check if the Wt mean activity uncertainty is at least $30 \%$ lower than the Wt mean Activity

\section{6) Nuclide MDA Report}


a. Check if, for the nuclides identified (marked with " + "), the Activity is higher than the Line MDA (Minimum detectable activity) for the evaluated lines (marked with "*")

7) Check how well data agrees with AES, Q-ICP-MS or MC-ICP-MS.

8) Check that data was uploaded correctly into LIMS and reflect "final" values.

a. Final values can be found on an Excel spreadsheet sent with the Apex-gamma report

9) Check that the data is consistent with what you know about the sample

a. Compare the half-life of the nuclide to the age of the sample, especially if it is an unusual analyte that is rarely or seldom found in any samples, or if there's no clear explanation as to how that analyte would be present in the sample

i. If the half-life is very short (days or months or even shorter) and the age of the sample is more than a year, unless there is another analyte in the sample that would decay into the nuclide in question, it's likely a misidentification.

b. If you can't answer this question, then find out more about the sample or compare with older results

10) Check that the data meets the requirements and goals of the customer

a. Did we meet detection limits?

b. Did we meet uncertainty requirements?

c. Did we measure and report all of the required data?

d. Did we measure analytes not requested by the customer? If so think about reporting them. 
Checklist for gas flow proportional counting data review:

\section{1) Check Sample ID}

2) Stack filters

a. Results should be close to the detector's background count rate (typically, gross beta is $<15$ DPM, and gross alpha is <5 DPM). Some stack filters are historically a little higher than others, so if in doubt check previous filters from the same facility.

b. For the FCF 8" filter, a count rate of ten times the sample blank requires either $\mathrm{Sr}-90$ or alpha spectrometry. Check that the results are reported in $\mu \mathrm{Ci}$.

3) $5 r-90$

a. Sheet "Final for C\#"

i. If the background count rate was manually subtracted, ensure that cell $\mathrm{J7}$ is 0 . Otherwise, the background will be subtracted twice from the gross count rate.

ii. Unless the Count time is something other than 30 minutes, the Elapsed Time should increase in 0.5 hour increments.

iii. Typically, the "Carrier NetWt" is on the order of 7-9.

b. Sheet "Sheet1"

i. The Sr-90 will be compared to the Cs-137 values here. Generally, samples with the highest Cs-137 activity will also have the highest Sr-90 activity.

ii. The percent recovery of the batch QC will be displayed in cell F7. Typically this is between 85 and $110 \%$.

iii. The activity of the blank run with the batch is displayed in cell E14. This value should be much lower than the samples in the batch (and is typically a similar order of magnitude for any sample blanks).

\section{4) Check how well data agrees with gamma, AES, Q-ICP-MS or MC-ICP-MS.}

a. For some samples, the $\mathrm{Sr}-90$ values will follow a similar pattern to the Cs-137 results, as the two can behave similarly under certain conditions.

5) Check that data was uploaded correctly into LIMS and reflect "final" values.

a. Final values can be found in the Excel spreadsheet under "Reccomended Reporting" or in "Sheet1".

b. Check that the uncertainty is at 2 sigma. The spreadsheet "Final for C\#" calculates a 1 sigma number, so a factor of 2 must be added in "Sheet1" prior to reporting.

6) Check that the data is consistent with what you know about the sample

a. If you can't answer this question, then find out more about the sample or compare with older results

7) Check that the data meets the requirements and goals of the customer

a. Did we meet detection limits?

b. Did we meet uncertainty requirements?

c. Did we measure and report all of the required data?

d. Did we measure analytes not requested by the customer? If so think about reporting them. 


\section{Checklist for HR-ICP-MS data:}

The instrument produces several reports, with different information in each of them. This checklist follows the numerical order of the reports.

1) Check Internal Standard Variation (Internal Standard Ratio file)

a. Large changes between samples and standards could suggest a problem with the sample matrix.

b. Large changes could also suggest a problem with the concentration of the internal standard added to the sample.

c. Large changes could suggest a problem with sample introduction system or other system instabilities.

2) Check calibration constant (Calibration data file)

a. Is the calibration at least qualitatively similar that observed from previous samples?

b. Are calibrations similar for different isotopes of the same element?

c. Some masses have isobaric interferences. Check that these masses were calibrated according to what the most likely/dominate species is in the sample.

3) Compare check standards to their known values

a. If values differ significantly from the expected value look at calibration curve.

4) Check Blanks: Dissolver blanks, Rinse and Instrumental blanks (Integrated file)

a. Look for count rates that are higher than what is typically expected for a particular mass.

b. In particular, inspect the count rates for evidence of contamination. The "light elements" $\mathrm{Cu}, \mathrm{Cr}$ and $\mathrm{Zn}$. Other elements of note are $\mathrm{Cd}, \mathrm{Hg}, \mathrm{Ba}$ and $\mathrm{Pb}$. $\mathrm{Zn}$ and Mo can sometimes be a concern as well.

5) Check concentrations in the dissolver blank (Concentrations file)

a. Concentrations should be near zero, at least within a few standard deviations of the blank concentration.

b. Negative values indicate a change in the background

c. If there is a significant value for the concentration of an analyte in the dissolver blank (either positive or negative) how does it compare to the concentration in the samples?

d. Large positive values could suggest "carry-over" from calibration standards.

6) Check sample and dissolver LIMS weights vs Excel spreadsheet

7) Check calculations

a. Are the correct cells referenced?

8) Check MDL

a. Based on the dilution factor and element is the MDL much larger or smaller than what would be expected based on past experience?

9) Check that duplicates match

a. If duplicates do not match is it for a particular analyte or is the difference consistent for all analytes in the run?

10) Check error 
a. Is the error much larger than what you would expect based on the concentration?

11) Perform a mass balance when appropriate

a. Are the concentrations of major species within what you would expect for the sample?

12) Check how well data agrees with ICP OES, Q-ICP-MS, MC-ICP-MS or gamma.

a. May not be available.

13) Check that data was uploaded correctly into LIMS and reflect "final" values.

14) Check that the data is consistent with what you know about the sample

a. If you can't answer this question then find out more about the sample.

b. For example, if the sample is irradiated fuel do the concentrations of fission products at least qualitatively track with the fission curve?

15) Check that the data meets the requirements and goals of the customer

a. Did we meet detection limits?

b. Did we meet uncertainty requirements?

c. Did we measure and report all of the required data?

d. Did we measure analytes not requested by the customer? If so think about reporting them. 
Checklist for ICP-OES data:

1) Ensure proper weights were entered for samples and dilutions

a. Make sure the UTEVA dilution factor of " 3 " was entered if a GPEC separation was performed prior to analysis

2) Compare "before" check standards to their known values

a. If values differ significantly from the expected values, check for trends to determine potential causes

3) Analyze "mid" and/or "after" check standards to determine if there was any significant instrumental drift

a. If drift is higher than the reported error for an analyte, increase the error for that analyte

4) If a GPEC separation was performed, check the spike recoveries to determine if the analytes of interest were recovered from the column

a. If values differ significantly from the expected values, check for trends to determine potential causes

5) Compare detection limit blank and dissolver blank values for any measurement above the method detection limit for that analyte

a. If the blank values are very similar, it is likely a result of the calibration curve

b. If blank values differ, it is likely due to process contamination

c. Blank corrections should not be made

i. Error can be increased to encompass a blank corrected value if needed

ii. If the blank value is a significant contributor to the measured value, make the highest number for that analyte a "<" (less than)

6) Ensure that the measured values are properly bracketed by the calibration standards

a. It is important to bracket as closely as reasonably possible on this instrument

7) If interference corrections were made, ensure that they make sense

a. Could it be contamination in the interference standard?

i. This is especially common for group 1 and group 2 elements

b. Are the blank values similar to the interferent value for the analyte in question?

i. If the interferent value is bracketed by the $D L$ before and $D L$ after values, it is likely noise, not a true interference

c. Does the correction result in a significant negative value?

d. Did the correction change the result more than $5 \%$ ?

8) If multiple lines were monitored for a single analyte, check that the line used in the final data was the best choice

a. Calibration, instrumental drift, MDL, spike recovery, and spectral interference should all be taken into account

9) Perform a mass balance when appropriate

a. Are the concentrations of major species within what you would expect for the sample?

10) Check how well data agrees with other analyses performed on the sample

11) Check that data was uploaded correctly into LIMS

12) Check that the data is consistent with what you know about the sample

a. If you can't answer this question then find out more about the sample

13) Check that the data meets the requirements and goals of the customer

a. Did we meet detection limits?

b. Did we meet uncertainty requirements? 
c. Did we measure and report all of the required data?

d. Did we measure analytes not requested by the customer? If so, think about reporting them. 
Checklist for liquid scintillation data review:

1) Check Sample ID

2) Check the tSIE value

a. Typically, the TSIE is between 200 and 800 . This is a parameter measured by the instrument, and is essentially a detection efficiency for tritium.

b. Even when tritium is not requested, tSIE values outside of this range can indicate the need to repeat the sample prep at a different dilution

\section{3) Tritium}

a. If tritium is requested, compare the CPMA and CPM B count regions.

i. A high CPMA along with a high CPMB will indicate the need to perform a distillation to separate the tritium from the rest of the components of the sample.

ii. A high CPMA compared to a CPMB that is close to the background may indicate the presence of tritium by itself. Historical knowledge of the sample type will indicate whether it is safe to assume the CPMA region is tritium or whether a distillation is required.

iii. If the activity for the CPMA region is below MDA, no distillation is required to the sample.

b. If distillation is required, check that the data has been forwarded as well. After distillation, the majority of the counts in the spectrum should lie in the CPMA (i.e. CPMB should be close to background). If this is not the case, the distillation may need to be repeated.

\section{4) Gross beta summing of regions CPMA and CPMB}

a. Check that the calculations report a sum of the two beta regions to reflect the total beta activity

b. The MDA should be a reflection of the sum of the two regions.

5) Check how well data agrees with gamma, AES, Q-ICP-MS or MC-ICP-MS.

a. For a gross beta measurement, typically the liquid scintillation results will be somewhere between a factor of 1-3 times the sum of the gamma results.

6) Check that data was uploaded correctly into LIMS and reflect "final" values.

a. Final values can be found in the Excel spreadsheet under "Reporting ALL in DPM".

7) Check that the data is consistent with what you know about the sample

a. If you can't answer this question, then find out more about the sample or compare with older results

8) Check that the data meets the requirements and goals of the customer

a. Did we meet detection limits?

b. Did we meet uncertainty requirements?

c. Did we measure and report all of the required data?

d. Did we measure analytes not requested by the customer? If so think about reporting them. 
Checklist for MC-ICP-MS data:

1) Raw data tab: check if there are at least two U500 U238/U235 results, as they are run in both the beginning and the end of each run.

a. The values should be around 1

b. Depending on the length of the run, more than two $U$ mass bias can be run. Ask the lead or analyst who performed the analysis for clarification

2) Raw data tab: check the 239/238. Should be small (around $10^{-5}$ )

3) U Bias Calculation tab:

a. Check if all the U500 U238/235 on the Raw data tab are being used

b. Check the formulas in all cells, and if the correct cell is referenced

c. Check the date of the analysis (as this can affect the corrected atom \% of the U500 standard)

d. Check if dilution factors are consistent for the Spike Dilution, Check Sample Dilution and Sample Information

e. Check if the Sample Weight and Dissolver Weight with the values on ALSTAR

4) Check sample tab:

a. The check sample depends on the type of sample that is being run. It should have similar ratios of $U$ as the sample (for example, SRMA for casting pins)

b. Check if the Atom $\%$ and Weight $\%$ are $100 \%$. If not, probably the wrong cells were referenced and should be checked

c. Check total U concentration, it should be what you expect for the used Check Sample. If not, probably the wrong cells were referenced and should be checked

5) Samples tab:

a. Check if the Atom $\%$ and Weight $\%$ are $100 \%$. If not, probably the wrong cells were referenced and should be checked

b. Check the formulas in some cells, and if the correct cell is referenced

6) Report tab:

a. Check if the correct cells are referenced

b. Check if any sample shows a different behavior, being a distinct iso\% or total U\%

7) Check how well data agrees with AES, Q-ICP-MS or gamma

8) Check that data was uploaded correctly into LIMS and reflect "final" values

9) Check that the data is consistent with what you know about the sample

a. If you can't answer this question, then find out more about the sample or compare with older results

10) Check that the data meets the requirements and goals of the customer

a. Did we meet detection limits?

b. Did we meet uncertainty requirements?

c. Did we measure and report all of the required data?

d. Did we measure analytes not requested by the customer? If so think about reporting them 
Checklist for Q-ICP-MS data:

1) Compare check standards to their known values

a. If values differ significantly from the expected value look at calibration curve.

2) Check calibration constant

a. Is the calibration at least qualitatively similar to that observed from previous samples?

b. Are calibrations similar for different isotopes of the same element?

c. Some masses have isobaric interferences. Check that these masses were calibrated according to what the most likely/dominate species is in the sample.

3) Check Blanks: Dissolver blanks, Rinse and Instrumental blanks

a. Look for count rates that are higher than what is typically expected for a particular mass.

b. In particular, inspect the count rates for evidence of contamination. The "light elements" $\mathrm{Cu}, \mathrm{Cr}$ and $\mathrm{Zn}$. Other elements of note are $\mathrm{Cd}, \mathrm{Hg}, \mathrm{Ba}$ and $\mathrm{Pb}$. $\mathrm{Zn}$ and Mo can sometimes be a concern as well.

4) Check concentrations in the dissolver blank

a. Concentrations should be near zero, at least within a few standard deviations of the blank concentration.

b. Negative values indicate a change in the background

c. If there is a significant value for the concentration of an analyte in the dissolver blank (either positive or negative) how does it compare to the concentration in the samples?

d. Large positive values could suggest "carry-over" from calibration standards.

5) Check Internal Standard Variation

a. Large changes between samples and standards could suggest a problem with the sample matrix.

b. Large changes could also suggest a problem with the concentration of the internal standard added to the sample.

c. Large changes could suggest a problem with sample introduction system or other system instabilities.

6) Check Sample and dissolver LIMS weights vs Excel spreadsheet

7) Check calculations

a. Are the correct cells referenced?

8) Check MDL

a. Based on the dilution factor and element, is the MDL much larger or smaller than what would be expected based on past experience?

9) Check that duplicates match

a. If duplicates do not match, is it for a particular analyte, or is the difference consistent for all analytes in the run?

10) Check error

a. Is the error much larger than what you would expect based on the concentration?

11) Perform a mass balance when appropriate

a. Are the concentrations of major species within what you would expect for the sample?

12) Check how well data agrees with AES, MC-ICP-MS or gamma.

a. May not be available.

13) Check that data was uploaded correctly into LIMS and reflect "final" values.

14) Check that the data is consistent with what you know about the sample

a. If you can't answer this question then find out more about the sample. 
b. For example, if the sample is irradiated fuel do the concentrations of fission products at least qualitatively track with the fission curve?

15) Check that the data meets the requirements and goals of the customer
a. Did we meet detection limits?
b. Did we meet uncertainty requirements?
c. Did we measure and report all of the required data?
d. Did we measure analytes not requested by the customer? If so, think about reporting them. 
Checklist for TIMS data:

1) Raw data tab: check if there are at least two U500 U238/U235 or CRM128 Pu242/Pu239 results, as they are run in both the beginning and the end of each run.

a. The values should be around 1

b. Depending on the length of the run, more than two $U$ or Pu mass bias can be run. Ask the lead or analyst who performed the analysis for clarification

2) U/Pu Bias Calculation tab:

a. Check if all the U500 U238/235 or CRM128 Pu242/Pu239 on the Raw data tab are being used

b. Check the formulas in all cells, and if the correct cell is referenced

c. Check the date of the analysis (as this can affect the corrected atom \% of the U500 and CRM 128 standard)

d. Check if dilution factors are consistent for the Spike Dilution, Check Sample Dilution and Sample Information

e. Check the Sample Weight and Dissolver Weight with the values on ALSTAR

3) Check sample tab:

a. The check sample depends on the type of sample that is being run. It should have similar ratios of $\mathrm{U}$ or Pu as the sample (for example, SRMA for casting pins or SNL for typical irradiated fuel)

b. Check if the Atom $\%$ and Weight $\%$ are $100 \%$. If not, probably the wrong cells were referenced and should be checked

c. Check total $\mathrm{U}$ or Pu concentration, it should be what you expect for the used Check Sample. If not, probably the wrong cells were referenced and should be checked

4) Samples tab:

a. Check if the Atom $\%$ and Weight $\%$ are $100 \%$. If not, probably the wrong cells were referenced and should be checked

b. Check the formulas in some cells, and if the correct cell is referenced

5) Report tab:

a. Check if the correct cells are referenced

b. Check if any sample shows a different behavior, being a distinct iso\% or total U\% or Pu\%

6) Check how well data agrees with AES, Q-ICP-MS or gamma

7) Check that data was uploaded correctly into LIMS and reflect final values

8) Check that the data is consistent with what you know about the sample

a. If you can't answer this question, then find out more about the sample or compare with older results

9) Check that the data meets the requirements and goals of the customer

a. Did we meet detection limits?

b. Did we meet uncertainty requirements?

c. Did we measure and report all of the required data?

d. Did we measure analytes not requested by the customer? If so think about reporting them 